\title{
Olfactory and rhinological evaluations in SARS-CoV-2 patients complaining of olfactory loss*
}

\author{
Giancarlo Ottaviano', Miryam Carecchio², Bruno Scarpa³, \\ Rosario Marchese-Ragona' \\ Department of Neurosciences, Otolaryngology Section, University of Padova, Padova, Italy \\ 2 Department of Neurosciences, Neurological Section, University of Padova, Padova, Italy \\ ${ }^{3}$ Department of Statistical Sciences and Department of Mathematics "Tullie Levi-Cijita", University of Padova, Padova, Italy
}

Rhinology 58: 4, 400 - 401, 2020

https://doi.org/10.4193/Rhin20.136

*Received for publication:

April 6, 2020

Accepted: April 24, 2020
Since December 2019, a novel coronavirus SARS-CoV-2 (Covid-19) outbreak emerged in China and spread rapidly in several countries. As of April 5, 2020, 1.218 .474 cases were confirmed with 65.884 deaths worldwide ${ }^{(1)}$.

The clinical manifestations of Covid-19 range from asymptomatic carrier status to severe pneumonia. In a study of 7,736 Covid-19 patients in China, of all the clinical symptoms, hyposmia was not reported in any patient ${ }^{(2)}$. Anyway, it is now clear that olfactory dysfunction may also be present in these patients ${ }^{(3)}$ as the only or prevalent manifestation ${ }^{(4)}$.

In the second week of March 2020 we evaluated a total of six Covid-19 patients complaining exclusively of sudden smell loss. All patients were either doctors or nurses at our hospital. In all of them SARS-CoV-2 was detected by real-time reverse transcriptase polymerase-chain-reaction (RT-PCR) assay from nasal/ throat swab specimens. All these cases were diagnosed during the same week. Five of them tested positive for SARS-CoV-2 due to close contact with a case of Covid-19, while one patient who had no contact with Covid-19 patients, asked to be tested after hearing of hyposmic colleagues positive for SARS-CoV-2. Only two patients reported myalgia the day before and one had both fever and cough few days after the onset of olfactory loss.

All the patients underwent (without the help of operators) the olfactory test "Le Nez du Vin"(5), which is a six supra-threshold odors forced multiple choice smell identification test. The odors presented were lemon, mint, strawberry, pine, vanilla and smoke ${ }^{(6)}$. The mean test result was 3.6 \pm 0.8 . As all subjects gave more than 2 wrong answers, Le Nez du Vin confirmed the olfactory loss in all of them ${ }^{(5)}$. Furthermore, the patients underwent some patient-reported outcome measures (PROMs) [Sino-nasal Outcome Test-22 (SNOT-22) and visual analog scale (VAS) for smell and taste $]^{(7)}$.
PROMs revealed significant alterations in the sense of smell and taste, while nasal symptoms other than olfaction/taste were found to be almost irrelevant ${ }^{(8)}$. Olfactory loss in patients with SARS-CoV-2 can be sensorineural, secondary to the involvement of the olfactory epithelium with the destruction of the olfactory sensory neurons, as hypothesized by Gane et al. ${ }^{(4)}$, or locally conductive, as recently reported by Eliezer and coworkers in a COVID-19 patient with sudden hyposmia who presented with CT and MRI evidence of bilateral inflammatory obstruction of the olfactory clefts without abnormalities of the bulbs and olfactory traits ${ }^{(9)}$.

After about 15 days, and three consecutive nasopharynx and throat swabs (>24 h intervals) negative for viral RNA, PROMs were repeated. We observed an overall improvement in both VAS (smell and taste) and SNOT 22, however all but one patient still complained of hyposmia and hypogeusia.

To contain the infection, it is extremely important to identify asymptomatic Covid-19 patients because this sub-population contributes to the spread of the disease. All individuals who complain of a sudden smell loss during a Covid-19 outbreak should undergo the Covid-19 diagnostic test.

Our results suggest that an overall improvement of olfaction starts in two weeks after the onset of smell loss. However, smell and taste alterations may persist even after the recovery from Covid-19 infection.

Key words: Covid-19, SARS-CoV-2, smell test, Nez du Vin, SNOT22, VAS, PROMs

Acknowledgement: Supported by a grant from Cariparo Foundation: "COVID-19-CNS understanding neurotropism and long-term brain damage from COVID-19."

\section{References}

1. Johns Hopkins Center for Systems Science and Engineering 2019-nCoV global cases. https://gisanddata.maps.arcgis.com/apps/ opsdashboard/index.html\#/bda7594740fd40299423467b48e9ecf6 consulted the 5th of april 2020

2. Guan WJ, Ni ZY, Hu Y, Liang WH, Ou CQ, He JX, et al. Clinical Characteristics of 
Coronavirus Disease 2019 in China. China Medical Treatment Expert Group for Covid19. N Engl J Med 2020, Feb 28. doi: 10.1056/ NEJMoa2002032

3. Giacomelli A, Pezzati L, Conti F, Bernacchia $D$, Siano M, Oreni L, et al. Self-reported olfactory and taste disorders in SARS-CoV-2 patients: a cross-sectional study. Clin Infect Dis. 2020 Mar 26. pii: ciaa330

4. Gane SB, Kelly C, Hopkins C. Isolated sudden onset anosmia in COVID-19 infection. A novel syndrome? Rhinology. 2020 Apr 2 doi: 10.4193/Rhin20.114.

5. McMahon C, Scadding GK. Le Nez du Vin-a quick test of olfaction. Clin Otolaryngol Allied Sci 1996;2:278-280

6. Ottaviano G, Zuccarello D, Frasson G, et al. Olfactory sensitivity and sexual desire in young adult and elderly men: an introductory investigation. Am J Rhinol Allergy 2013;27:157-161

7. Rimmer J, Hellings P, Lund VJ, Alobid I, Beale T, Dassi C, et al. European position paper on diagnostic tools in rhinology. Rhinology 2019;57(Suppl S28):1-4

8. Pendolino AL, Scarpa B, Ottaviano G. Relationship Between Nasal Cycle, Nasal Symptoms and Nasal Cytology. Am J Rhinol Allergy 2019;33:644-649

9. Eliezer, M., et al., Sudden and Complete Olfactory Loss Function as a Possible Symptom of COVID-19. JAMA Otolaryngol Head Neck Surg. 2020 Apr 8. doi: 10.1001/ jamaoto.2020.0832.
Giancarlo Ottaviano, MD, PhD.

Department of Neurosciences

Otolaryngology Section

University of Padova

Via Giustiniani 2

35128 Padova

Italy

Tel. +39 (0)498214470

Fax: +39 (0)498213113

E-mail: giancarlo.ottaviano@unipd.it 